\title{
Study for the discrepancy of arterial blood pressure in accordance with method, age, body part of measurement during general anesthesia using sevoflurane
}

\author{
Ji Ho Lee ${ }^{1}$, Joung-Min Kim², Ki Ryang Ahn², Chun Sook Kim², Kyu Sik Kang ${ }^{2}$, Jin Hun Chung ${ }^{2}$, Ji-Won \\ Chung $^{2}$, and Sie Hyeon Yoo ${ }^{2}$ \\ ${ }^{1}$ Department of Occupational and Environmental Medicine, College of Medicine, Ulsan University Hospital, Ulsan, ${ }^{2}$ Department of \\ Anesthesiology and Pain Medicine, College of Medicine, Soonchunhyang University, Cheonan, Korea
}

Background: Many pieces of previous research on measuring blood pressure (BP) using different methods focused on the disparity in the results. However, none of them dealt with the disparity caused by the difference in age and inhalation anesthetics. We attempted to find the variance in accordance with age, body part, and measuring methods (invasive vs noninvasive) and also studied how sevoflurane influences BP as the operation progresses.

Methods: In sixty patients, we measured the arterial BP in the upper and lower limbs by noninvasive methods before inducing anesthesia. After induction, we used sevoflurane to maintain anesthesia, and injected catheters into the radial artery and dorsalis pedis artery to measure arterial pressure at every ten minute by both invasive and noninvasive methods.

Results: The patients who were 40 or older showed significantly higher values in the systolic BP than the patients younger than 40. The values of systolic and diastolic BP measured by a noninvasive oscillometric method were meaningfully higher than those measured by an invasive method. As the operations progressed, the lower limbs showed higher systolic pressure than the upper limbs regardless of measuring methods, whereas the opposite is true for diastolic pressure.

Conclusions: The values in the arterial BP were measured high by noninvasive method. Systolic BP were estimated significantly high in the older patients and in the lower leg. Due to the effect of sevoflurane, the diastolic BP in the lower limbs becomes lower than that of upper limbs regardless of measuring methods, as the operation progresses. (Korean J Anesthesiol 2011; 60: 323-328)

Key Words: Blood pressure, Dorsalis pedis artery, Radial artery, Sevoflurane.

Received: July 20, 2010. Revised: 1st, October 6, 2010; 2nd, November 16, 2010. Accepted: November 20, 2010.

Corresponding author: Sie Hyeon Yoo, M.D., Department of Anesthesiology and Pain Medicine, College of Medicine, Soonchunhyang University, 23-20, Byeongmyeong-dong, Dongnam-gu, Cheonan 330-721, Korea. Tel: 82-41-570-2827, Fax: 82-41-573-3559, E-mail: doc0126@ dreamwiz.com

(c) This is an open-access article distributed under the terms of the Creative Commons Attribution Non-Commercial License (http:// creativecommons.org/licenses/by-nc/3.0/), which permits unrestricted non-commercial use, distribution, and reproduction in any medium, provided the original work is properly cited. 


\section{Introduction}

Under general anesthesia, hemodynamometry (blood pressure monitoring) is an essential element for monitoring patients. However, the arterial pressure value can vary depending on many factors, such as the measurement method, measuring location, posture, length and diameter of catheter, type of monitor, patient status, and drug dosage [1,2]. Arterial pressure is measured with invasive or non-invasive methods under anesthesia, but in most cases, these 2 methods produce different values due to diverse variables [3]. Comparison of arterial blood pressure by direct or indirect sphygmomanometry has been a topic of study for decades; nevertheless, a conclusion cannot be reached as the targets of measurement of the 2 methods are essentially different $[4,5]$. In clinical cases, whether both methods of sphygmomanometry are used at the same time or just one, it is important for anesthesiologists, who need to manage patients, to trust the outcome. Thus, it is important for them to consider and have knowledge of patterns of differences between measurement methods, even if they cannot make these comparisons themselves.

Differences in blood pressure according to the location where the measurement is taken are as follows: systolic pressure increases as the measuring location is moved toward the periphery of the body away from the heart; diastolic pressure does not differ. There is also a deformity in the arterial pressure waveform due to peripheral vascular resistance and reflection; this is severe in elderly patients [3,6]. However, not enough reviews have been performed to reach a conclusion on how trustworthy values measured from the lower limbs of elderly patients or of those who suffer from high blood pressure, even though differences in these values compared with non-invasive measurements can influence the addition of a vasopressor or anesthetic. Sevoflurane is a commonly used inhalation anesthetic that decreases autonomic reflection and heart rate variations and lowers systemic vascular resistance $[7,8]$. Many reports on hemodynamic changes of sevoflurane after induction of anesthesia have been published; yet, there have been no comparative studies of hemodynamometry as the operation progresses. This study aimed to determine how differences in age, measuring location, measurement method, and use of sevoflurane influence blood pressure as an operation progresses by invasively measuring the radial artery and dorsalis pedis artery while simultaneously measuring the blood pressure of the upper and lower limbs using a non-invasive method.

\section{Meterials and Methods}

This study was approved by the Institutional Review Board and informed consent was obtained from the patients. Sixty patients (35 male and 25 female) aged 20-78 years who were about to undergo operations for facial fracture between January 2009 and December 2009, and who were rated as level 1 or 2 according to the American Society of Anesthesiologists classification, were enrolled. Obese patients with a body mass index (BMI) $>35$, and those who suffered from high blood pressure, cardiovascular diseases, or diabetes were excluded. For premedication, all patients received intramuscular (IM) midazolam $3 \mathrm{mg}$ and glycopyrrolate $0.2 \mathrm{mg}$ half an hour before anesthesia induction. After transferring patients to the operating room, they were attached to standard monitors, such as an electrocardiogram, $\mathrm{SpO}_{2}$ monitor, and a sphygmodynamometer. The patients were given 10 minutes to stabilize before noninvasive arterial pressure from the humerus of both arms and both the lower legs was measured and recorded via 2 monitors (Hewlett Packard-6M1046, 140 Herrenberger strasse, Böblingen, Germany). After a propofol injection, the patients were administered IV $1 \%$ lidocaine $4 \mathrm{ml}$ to reduce pain followed by propofol $2 \mathrm{mg} / \mathrm{kg}$ to induce unconsciousness. Rocuronium 0.6 $\mathrm{mg} / \mathrm{kg}$ was then injected to facilitate endotracheal intubation, and vital signs were observed. Patients were kept under anesthesia with sevoflurane at a rate between 1.8 and 2.2 vol\%. After anesthesia induction, to make measurement easier, the upper and lower limbs on the same side as the anesthesiologists were selected for convenience so they could continue to measure arterial pressure by catheterizing a $22 \mathrm{G}$ catheter in the radial artery at the wrist and the dorsalis pedis artery at the instep. Blood pressure cuffs for the upper (humerus) and lower limbs (ankle) were used to simultaneously measure arterial pressure non-invasively. After all preparations, and once hemodynamic changes stabilized, both upper and lower limbs were measured and recorded with invasive and noninvasive methods (T0). Blood pressure was measured 4 times in total (T10, T20, T30, T40), whenever the patient was stabilized in terms of hemodynamics; patients were not touched and the operating table was not moved so as to minimize errors caused by movement or differences in reference point.

Student's t-test and analysis of variance (ANOVA) were used to compare patients' systolic and diastolic blood pressure based on gender, age group, and BMI. Since comparisons of blood pressure using invasive and non-invasive methods, and the arterial pressure of the upper and lower limbs, were taken from 1 patient, the results were not independent. Thus, a repeated measures ANOVA was used. We used SPSS (version 14.0 Korea) for all statistical analyses. Every variation is indicated as \pm standard deviation. P values $<0.05$ were considered statistically significant.

\section{Results}

Mean age of patients was 42.5 (range, 20-78 years). For 
blood pressure measurements in the upper and lower limbs on both sides non-invasively measured before anesthesia, the systolic pressure of the lower limb was significantly higher ( $\mathrm{P}$ $<0.001)$ than that of the ipsilateral upper limb, but there was no significant difference in diastolic pressure (Table 1). Gender did not influence measured arterial pressure. The patient group with a BMI $<22.9$ did not differ in terms of systolic blood pressure, but diastolic blood pressure was notably lower ( $\mathrm{P}<0.05$, post hoc) when they were compared with the $\geq 25$ BMI group. Systolic pressure of patients who were more than 40 years old was significantly higher compared with that of patients younger than 40 years $(\mathrm{P}<0.05)$; however, diastolic blood pressure was not influenced by age, and there was also no contrast between the blood pressure of the upper and lower limbs (Table 2).
When arterial pressure based on the measuring location and method was compared, the systolic blood pressure of the lower limbs measured right after anesthesia induction (T0) was significantly higher than the systolic pressure of the upper limbs for both measuring methods $(\mathrm{P}<0.05)$. For diastolic blood pressure, there was no variation in values measured by both invasive and non-invasive methods in the upper limbs; however, values measured by the invasive method in the lower limbs were significantly lower than non-invasively measured values $(\mathrm{P}<0.05)$. For diastolic arterial pressure, there was no significant difference between the upper and lower limbs, and invasively measured blood pressure was significantly lower than non-invasively measured blood pressure for both the upper and limbs $(\mathrm{P}<0.05)$ (Table 3).

Comparisons of methods for measuring blood pressure in

Table 1. Baseline Blood Pressures Before Induction

\begin{tabular}{|c|c|c|c|c|c|c|c|}
\hline \multicolumn{4}{|c|}{ Systolic blood pressure (mmHg) } & \multicolumn{4}{|c|}{ Diastolic blood pressure (mmHg) } \\
\hline Rt. arm & Lt. arm & Rt. leg & Lt. leg & Rt. arm & Lt. arm & Rt. leg & Lt. leg \\
\hline $132.8 \pm 2.1$ & $133.6 \pm 2.2$ & $152.0 \pm 2.2^{*}$ & $154.8 \pm 2.4^{*}$ & $80.1 \pm 1.7$ & $82.6 \pm 1.6^{\dagger}$ & $80.6 \pm 1.7$ & $82.4 \pm 1.5$ \\
\hline
\end{tabular}

Values are estimated mean \pm SE adjusted by BMI and age. $* \mathrm{P}<0.05$ taken by general linear model, compared leg with arm. ${ }^{\dagger} \mathrm{P}<0.05$ compared left with right arm.

Table 2. Blood Pressures before Induction Considering Demographic Factors

\begin{tabular}{|c|c|c|c|c|c|c|c|c|}
\hline & \multicolumn{4}{|c|}{ Systolic blood pressure (mmHg) } & \multicolumn{4}{|c|}{ Diastolic blood pressure (mmHg) } \\
\hline & Rt. arm & Lt. arm & Rt. leg & Lt. leg & Rt. arm & Lt. arm & Rt. leg & Lt. leg \\
\hline \multicolumn{9}{|l|}{ Sex } \\
\hline M & $136.4 \pm 14.2$ & $136.7 \pm 14.3$ & $154.8 \pm 16.4$ & $159.5 \pm 17.2$ & $81.7 \pm 11.7$ & $84.4 \pm 11.7$ & $83.2 \pm 12.9$ & $86.0 \pm 12.0^{\dagger}$ \\
\hline $\mathrm{F}$ & $128.2 \pm 19.4$ & $131.5 \pm 21.2$ & $149.7 \pm 20.1$ & $149.8 \pm 20.7$ & $78.0 \pm 10.2$ & $80.4 \pm 10.0$ & $77.8 \pm 9.7$ & $77.8 \pm 7.5$ \\
\hline \multicolumn{9}{|l|}{ Age (yr) } \\
\hline$<39$ & $128.5 \pm 15.0^{*}$ & $129.2 \pm 14.2 *$ & $148.0 \pm 14.7^{*}$ & $152.2 \pm 13.7$ & $79.6 \pm 12.2$ & $82.9 \pm 12.5$ & $81.4 \pm 12.7$ & $84.4 \pm 11.6$ \\
\hline$>40$ & $138.5 \pm 17.2$ & $140.7 \pm 18.4$ & $158.1 \pm 19.7$ & $159.4 \pm 23.1$ & $81.0 \pm 10.7$ & $82.9 \pm 9.8$ & $81.0 \pm 11.4$ & $81.3 \pm 10.7$ \\
\hline \multicolumn{9}{|l|}{ BMI } \\
\hline$<22.9$ & $128.3 \pm 19.4$ & $130.4 \pm 18.2$ & $148.0 \pm 16.6$ & $152.7 \pm 20.8$ & $74.4 \pm 10.3^{\dagger}$ & $79.0 \pm 10.9$ & $75.7 \pm 10.9^{\ddagger}$ & $79.7 \pm 10.7$ \\
\hline $23-24.9$ & $135.3 \pm 14.3$ & $137.5 \pm 17.6$ & $154.8 \pm 19.9$ & $157.1 \pm 18.7$ & $82.9 \pm 10.6$ & $84.3 \pm 10.3$ & $84.4 \pm 12.3$ & $84.9 \pm 13.0$ \\
\hline$>25$ & $137.8 \pm 14.0$ & $137.7 \pm 15.4$ & $157.2 \pm 17.0$ & $158.2 \pm 17.4$ & $85.7 \pm 10.5$ & $86.6 \pm 11.3$ & $85.1 \pm 11.0$ & $85.1 \pm 9.3$ \\
\hline
\end{tabular}

Values are mean \pm SD and measured by noninvasive method. BMI: body mass index (weight/[height $\left.]^{2}\right)$. $\mathrm{P}<0.05$ taken by t-test, compared younger with older age group (older than 40 ). ${ }^{\dagger} \mathrm{P}<0.05$ compared male with female. ${ }^{\dagger} \mathrm{P}<0.05$ compared lower with higher BMI groups (BMI $>23$ ).

Table 3. Comparisons of Blood Pressure by Noninvasive and Invasive Measuring Methods

\begin{tabular}{lccccc}
\hline & \multicolumn{2}{c}{ Systolic blood pressure $(\mathrm{mmHg})$} & & \multicolumn{2}{c}{ Diastolic blood pressure (mmHg) } \\
\cline { 2 - 3 } \cline { 5 - 6 } & Upper arm & Lower leg & & Upper arm & Lower leg \\
\hline Before Induction by noninvasive method & $132.0 \pm 2.0$ & $152.5 \pm 2.5^{*}$ & & $79.8 \pm 1.6$ & $80.8 \pm 1.7$ \\
After induction (T0) by noninvasive method & $107.7 \pm 1.8$ & $120.3 \pm 2.1^{*}$ & & $70.2 \pm 1.6$ & $70.8 \pm 1.9$ \\
Invasive method & $107.2 \pm 2.4$ & $114.8 \pm 2.5^{*, \dagger}$ & & $64.9 \pm 1.5^{\ddagger}$ & $63.1 \pm 1.7^{\ddagger}$ \\
\hline
\end{tabular}

Values are estimated mean \pm SE adjusted by BMI andage. Values were measured by invasive method at radial artery or dorsalis pedis artery. ${ }^{*} \mathrm{P}<0.05$ compared lower leg with upper arm. ${ }^{\dagger} \mathrm{P}<0.05$ compared invasive with noninvasive method. ${ }^{\dagger} \mathrm{P}<0.05$ compared invasive with noninvasive method. T0: right after induction. 
Table 4. Blood Pressure During the Operation Using Sevoflurane

\begin{tabular}{|c|c|c|c|c|c|c|c|c|}
\hline & \multicolumn{4}{|c|}{ Systolic Blood Pressure (mmHg) } & \multicolumn{4}{|c|}{ Diastolic Blood Pressure (mmHg) } \\
\hline & \multicolumn{2}{|c|}{ Noninvasive method } & \multicolumn{2}{|c|}{ Invasive method } & \multicolumn{2}{|c|}{ Noninvasive method } & \multicolumn{2}{|c|}{ Invasive method } \\
\hline & Arm & Leg & Arm & Leg & Arm & Leg & Arm & Leg \\
\hline Time 10 & $107.8 \pm 1.9$ & $120.5 \pm 2.0^{*}$ & $107.3 \pm 2.5$ & $114.9 \pm 2.5^{*, \dagger}$ & $70.3 \pm 1.6$ & $70.8 \pm 1.9$ & $64.7 \pm 1.5^{\dagger, \S}$ & $63.0 \pm 1.6^{\dagger}$ \\
\hline Time 20 & $106.2 \pm 2.5$ & $119.0 \pm 2.5^{*}$ & $104.8 \pm 2.6^{\dagger}$ & $110.6 \pm 2.9^{*, \dagger}$ & $67.0 \pm 1.6$ & $67.7 \pm 1.8$ & $64.6 \pm 2.0^{\dagger, \S}$ & $58.9 \pm 1.6^{\dagger}$ \\
\hline Time 30 & $108.6 \pm 2.2$ & $121.9 \pm 2.5^{*}$ & $105.5 \pm 2.4^{\dagger}$ & $110.5 \pm 2.4^{*, \dagger}$ & $71.4 \pm 1.8^{\ddagger}$ & $69.3 \pm 2.0$ & $64.1 \pm 1.7^{\dagger, \S}$ & $61.2 \pm 1.5$ \\
\hline Time 40 & $107.0 \pm 2.3$ & $119.9 \pm 2.4^{*}$ & $101.4 \pm 2.2^{\dagger}$ & $104.9 \pm 2.4^{*, \dagger}$ & $68.3 \pm 1.8^{\ddagger}$ & $67.5 \pm 1.7$ & $61.0 \pm 1.5^{\dagger, \S}$ & $58.6 \pm 1.5^{\dagger}$ \\
\hline
\end{tabular}

Values are estimated mean \pm SE adjusted by BMI and age. $* \mathrm{P}<0.05$ taken by general linear model. Values were measured by invasive method at radial artery or dorsalis pedis artery. ${ }^{*} \mathrm{P}<0.05$ leg compared with $\operatorname{arm} .{ }^{\dagger} \mathrm{P}<0.05$ compared invasive with noninvasive method in arm. ${ }^{\dagger} \mathrm{P}<0.05$ compared invasive with noninvasive method in leg. ${ }^{\S} \mathrm{P}<0.05$ compared arm with leg by invasive method. T10, T20, T30, T40: mean 10 minutes, twenty minutes, thirty minutes and forty minutes after induction, respectively.

the upper and lower limbs during the operation (T10, T20, T30, T40) showed that systolic blood pressure measured by the non-invasive method was generally higher than that measured by the invasive method ( $\mathrm{P}<0.05)$, and, except for the initial measurement (T10), differences in blood pressure due to the measuring method were significant $(\mathrm{P}<0.05)$. In addition, blood pressure in the lower limbs was higher than that in the upper limbs, and this difference was significant for both noninvasive and invasive methods $(\mathrm{P}<0.05)$; the value was similar to that of measurements taken before the operation began. Even for diastolic blood pressure, values measured by the noninvasive method were generally higher than those measured by the invasive method $(\mathrm{P}<0.05)$. As the operation began and progressed, diastolic arterial pressure of the upper and lower limbs measured by the non-invasive method differed from the systolic pressure-blood pressure in the upper limbs in the latter period (T30, T40) was significantly higher than in the lower limbs, and invasively measured blood pressure in the upper limbs was significantly higher than in the lower limbs at all measurement times (T10-T40) $(\mathrm{P}<0.05)$ (Table 4).

\section{Discussion}

Arterial pressure is lateral wall pressure applied to the arteries due to blood flow $[9,10]$. Both non-invasive automatic and invasive sphygmomanometry reflect the effects of all fluids; in other words, the addition of pressure as well as the kinetic and potential energy of blood that reaches the vessel wall or catheter is calculated. For example, in the invasive method, the speed of blood entering the catheter will become 0 so kinetic energy will be lost; however, due to the principle of conservation of energy, it will be converted to pressure energy. Thus, if both the non-invasive and invasive methods are precisely measured at 1 location without any errors, outcomes from direct measurement will always be larger [9]. Because of this, the 2 measurement methods are dissimilar so it is not appropriate to compare the accuracy of measured arterial pressure, and it is also meaningless to try decrease errors between the two $[4,5]$. However, in reality, there are cases when we have to compare values measured by both direct and indirect measurement methods, or to focus on one of the methods as a reference to manage blood pressure. Sometimes, we see broader differences in measured values than expected.

We have proposed 3 hypotheses, which are as follows: first, blood pressure in older patients will be higher than that in younger patients, and differences in arterial pressure between the upper and lower limbs will also be higher; second, as blood pressure values in the upper and lower limbs measured by the indirect method are higher in the lower limbs, values from direct measurement will also be higher in the lower limbs; and third, since the influence of sevoflurane, which is an inhalation anesthetic, decreases in both the upper and lower limbs, differences in blood pressure in both the upper and lower limbs will remain as anesthesia progresses.

As we move from the heart to the periphery, reflection waves from the periphery distort the waveform of blood pressure as it moves to the periphery. As a result, contractile pressure and pulse pressure values appear higher and the waveform of the relaxation phase is lost [11]. In this study, contractile pressure in the lower limbs measured by the non-invasive method before the induction of anesthesia was significantly higher than that in the upper limbs. This was greater in elderly patients, as the compliance of the artery is lower in the elderly compared with younger patients; we also witnessed a relevant increase in contractile pressure in older patients during this study. However, we could not find any important variations in diastolic blood pressure between the under-40 and over-40 age groups (Table 2).

Gender and BMI did not influence blood pressure; the group with a BMI less than 22.9 showed significantly lower diastolic blood pressure than the other groups due to the same cuff being used for measurements in all patients (i.e., since the width of 
the cuff was the same, the results were relatively lower; Table 2 ). We have to consider errors arising from the cuff on either very skinny or obese patients, and we should focus on the reappearance of measured values rather than on the accuracy of the measurement.

From the study outcomes, among blood pressure measurements performed right after the induction of anesthesia, noninvasively measured blood pressure was significantly higher than invasively measured blood pressure, except for systolic blood pressure in the upper limbs (Table 3). Interestingly, diastolic blood pressure did not vary between the upper and lower limbs with both measurement methods; yet, as anesthesia progressed, diastolic pressure in the lower limbs was lower than that in the upper limbs with both measurement methods (Table 4). Taking these results into account, arterial pressure of the dorsalis pedis artery will be higher when we invasively measure blood pressure in both the radial and dorsalis pedis arteries; thus, if we only monitor the dorsalis pedis artery with the invasive method, we might fail to notice the low blood pressure. These results contrast with those previously reported [12-14], and we therefore compared non-invasively measured values from the upper limbs. The reason for the discrepancy may be the effect of vasodilation, which is caused by decreased angiokinetic control, and lower vascular resistance in the whole body resulting from sevoflurane during anesthesia; the effects are greater in the lower limbs since these have a larger vascular bed in the measured location. Medications that are applied to other parts of the body-i.e., not the measuring locations-can influence hemodynamometry and, as with other anesthetics, sevoflurane can directly affect the heart and vascular smooth muscles, and indirectly affect the autonomic nervous system. Sevoflurane can therefore induce antihypertensive effects, depending on the amount used [7,15-18]. In this study, as the operation progressed, diastolic pressure of both the upper and lower limbs reversed due to the effect of vasodilation and decreased peripheral vascular resistance resulting from the inhalation anesthetic. However, further studies are needed to determine why the same effect is not exerted on systolic pressure. Of course, when we manage patients in clinics, diastolic pressure is not more important than systolic pressure; nonetheless, if diastolic pressure is significantly lower or higher, we should double check the result with other measurement methods.

Comparison of the radial and dorsalis pedis arteries by direct measurement has been reported in various studies $[19,12,14]$. Among these, research conducted by Abou-Madi et al. [19] showed that the measured values of the dorsalis pedis artery were higher than those of the radial artery when anesthesia was induced by fentanyl, thiopental sodium, and sodium nitroprusside; however, when anesthesia was induced by isoflurane, pressure in the radial artery was significantly higher. The differences in direct and indirect measurement methods were first compared by Van Bergen et al. in 1954 [20], when he discussed variation of blood pressure based on measurement method. Since then, a few decades have passed and we have witnessed the development of transducers and recorders, but the problem has not been completely solved. Physiological dissimilarity of patients is one cause of differences between the 2 measurement methods; however, in most cases, they depend on the frequency responses of the recording system [3]. Previously, we stated that comparing the accuracy of the 2 methods is inappropriate, but in many cases, they are compared clinically. Even in these cases, we refer to a tendency toward differences rather than to differences in the accuracy of the measured values. With most patients, systolic pressure measured by the non-invasive method will be placed within $\pm 10 \mathrm{mmHg}$ of directly measured values if the circumference of the upper limb is more than $35 \mathrm{~cm}$ or under $24 \mathrm{~cm}$, or if the patients do not suffer from aortic insufficiency [3]. In addition, diastolic pressure measured by the traditional Riva-Rocci/Korotkoff technique is generally higher than arterial pressure, which is measured directly [3]. Even in this study, values measured using the noninvasive method were generally higher than values measured using the invasive method; however, since there are many interrelated factors, we cannot clearly explain this. Clinically, diastolic pressure of lower limbs may be lower than that of upper limbs when general anesthesia is induced using sevoflurane; thus, if a diastolic pressure measurement is low, rather than administrating pressure agents by assuming that the pressure of the lower limbs will be lower, we should measure the upper limbs to make a comparison and thereby stop the unnecessary administration of pressure agents.

The low number of patients in this study was not its only limitation-it is also possible that we would obtain different results if we used different non-invasive measurement machines. In other words, non-invasively measured outcomes that are evaluated with a certain machine cannot be compatible with other machines. In addition, although we made an effort to measure different values simultaneously, there was likely some kind of time lag, even if of a few seconds, between the invasive and non-invasive measurement methods. Further studies of changes in hemodynamometry caused by operations using other anesthetics, various pressure agents, and vasodepressors are also necessary.

In conclusion, systolic blood pressures of the older patients, the lower limbs and noninvasive measurements showed significantly higher than those of the younger patients, the upper limb and invasive measurement. Also, the effects of sevoflurane were greater at the diastolic blood pressure in the lower limbs as an operation progressed. 


\section{References}

1. Mason DE, Brown MJ. Monitoring of anesthesia. In: Anesthesia and Analgesia in Laboratory Animals. Edited by Kohn DF, Wixson SK, White WJ, Benson GJ: London, Academic Press. 1997, pp 73-82.

2. van Egmond J, Hasendbos M, Crul JF. Invasive vs non-invasive measurement of arterial pressure. Br J Anaesth 1985; 57: 434-44.

3. Bruner JM, Krenis LJ, Kunsman JM, Sherman AP. Comparison of direct and indirect methods of measuring arterial blood pressure, part III. Med Instrum 1981; 15: 182-8.

4. Raftery EB. Direct versus indirect measurement of blood pressure. J Hypertens Suppl 1991; 9: S10-2.

5. Ahn W, Jung CW. A comparison of the Vasotrac with invasive arterial blood pressure monitoring. Anesth Analg 2006; 102: 333.

6. O'Rourke MF, Blazek JV, Morreels CL Jr, Krovetz LJ. Pressure wave transmission along the human aorta. Changes with age and in arterial degenerative disease. Circ Res 1968; 23: 567-79.

7. Ebert TJ. Cardiovascular and autonomic effects of sevoflurane. Acta Anaesthesiol Belg 1996; 47: 15-21.

8. Ebert TJ, Harkin CP, Muzi M. Cardiovascular responses to sevoflurane: a review. Anesth Analg 1995; 81: S11-22.

9. Nichols WW, O'Rourke MF. McDonald's blood flow in arteries: theoretical, experimental and clinical principles. 5th ed. New York, Oxford University Press. 2005, pp 22-4.

10. Lee JE. Cardiovascular monitoring. In: Anesthesia and Pain Management. 2nd ed. Edited by Korean Society of Anesthesiologists. Seoul, Elsevier Korea LLC. 2009, pp 821-2.

11. Darovic GO, Vanriper S. Aterial pressure recording. In: Hemodynamic monitoring. 2nd ed. Edited by Darpvoc GP: Philadelphia, WB Saunders. 1995, pp 177-210.
12. Parry T, Hirsch N, Fauvel N. Comparison of direct blood pressure measurement at the radial and dorsalis pedis arteries during surgery in the horizontal and reverse Trendelenburg positions. Anaesthesia 1995; 50: 553-5.

13. Johnstone RE, Greenhow DE. Catheterisation of the dorsalis pedis artery. Anesthesiology 1973; 39: 654-5.

14. Spoerel WE, Deimling P, Aitken R. Direct arterial pressure monitoring from the dorsalis pedis artery. Can Anaesth Soc J 1975; 22: 91-9.

15. Crawford MW, Lerman J, Saldivia V, Carmichael FJ. Hemodynamic and organ blood flow responses to halothane and sevoflurane anesthesia during spontaneous ventilation. Anesth Analg 1992; 75 : 1000-6.

16. Ebert TJ, Muzi M. Sympathetic hyperactivity during desflurane anesthesia in healthy volunteers. A comparison with isoflurane. Anesthesiology 1993; 79: 444-53.

17. Ebert TJ, Muzi M, Lopatka CW. Neurocirculatory responses to sevoflurane in humans. A comparison to desflurane. Anesthesiology 1995; 83: 88-95.

18. Bernard JM, Wouters PF, Doursout MF, Florence B, Chelly JE, Merin RG. Effects of sevoflurane and isoflurane on cardiac and coronary dynamics in chronically instrumented dogs. Anesthesiology 1990; 72: 659-62.

19. Abou-Madi M, Lenis S, Archer D, Ravussin P, Trop D. Comparison of direct blood pressure measurements at the radial and dorsalis pedis arteries during sodium nitroprusside-and isofluraneinduced hypotension. Anesthesiology 1986; 65: 692-5.

20. VAN BERGEN FH, WEATHERHEAD DS, TRELOAR AE, DOBKIN $\mathrm{AB}$, BUCKLEY JJ. Comparison of indirect and direct methods of measuring arterial blood pressure. Circulation 1954; 10: 481-90. 